\title{
Comparative genomic hybridization for the diagnosis of melanoma
}

\author{
Christopher Vanison • Neil Tanna • Ananth S. Murthy
}

Received: 28 March 2009 / Accepted: 27 October 2009 / Published online: 9 December 2009

(C) The Author(s) 2009. This article is published with open access at Springerlink.com

\begin{abstract}
Despite advancements in protocols, a subset of melanocytic lesions continues to pose diagnostic challenges. This is particularly true in the pediatric population where certain congenital nevi mimic melanoma. Recently, comparative genomic hybridization (CGH) has been utilized to support diagnoses of melanocytic lesions based on DNA copy number changes. Because distinct differences in copy number changes have been shown to occur in malignant melanoma and benign nevi, CGH can be a useful adjunct when diagnosis based on histology alone is indeterminate. The authors discuss the benefits of using CGH to aid in the diagnosis of melanocytic lesions that are difficult to characterize as malignant or benign based on clinical and histologic features alone. This paper presents a brief clinical report and review of the literature. A 13-yearold Caucasian male presented to an academic tertiary care medical center after a shave biopsy unexpectedly revealed malignant melanoma with positive deep margins. Following complete excisional biopsy, the diagnosis of malignant melanoma with depth of $0.92 \mathrm{~mm}$ was confirmed, both by
\end{abstract}

Oral presentation at Aesthetic Facial Reconstruction in Adults and Children Symposium, San Diego, CA, USA on February 19, 2009.

C. Vanison $\cdot$ N. Tanna $(\bowtie)$

Division of Otolaryngology-Head \& Neck Surgery,

The George Washington University,

2150 Pennsylvania Ave., NW,

Washington, DC 20037, USA

e-mail: ntanna@gmail.com

\section{A. S. Murthy}

Division of Plastic Surgery, Children's National Medical Center, Washington, DC 20037, USA

\section{A. S. Murthy}

Division of Plastic Surgery, The George Washington University, Washington, DC 20037, USA the home institution's pathologist and by consultant dermatopathologists at two separate academic tertiary medical centers. Sentinel lymph node biopsy revealed a small focus of metastatic melanoma, this lead to a left-sided modified radical neck dissection. All nodes removed were negative for disease, and surgical and postsurgical care was uncomplicated. Before proceeding with interferon therapy, CGH was performed on the tissue from the primary lesion. Other than a slight amplification of chromosome 16p, no other aberrations were detected favoring a benign lesion. Ultimately, the diagnosis was amended to compound melanocytic nevus of the nose with benign nevus cell rest in the sentinel node. While histopathologic evaluation is the current gold standard for the diagnosis of melanoma, there are many cases where it is inaccurate. The use of CGH in the evaluation of histologically equivocal lesions may allow certain patients to avoid invasive procedures and associated morbidities. The authors propose that, in these select diagnostically challenging cases, tissue analyses by CGH may be beneficial before proceeding to more invasive procedures such as sentinel node biopsy and complete lymphadenectomy.

Keywords Melanoma $\cdot$ Diagnosis $\cdot$ Nevus $\cdot$ Nevi . Comparative genomic hybridization

\section{Introduction}

Current literature demonstrates poor concordance among pathologists in diagnosing malignant melanoma $[1,2]$. This may be particularly true in the pediatric population where certain melanocytic lesions such as Spitz nevi may be impossible to differentiate from malignant melanoma based on histology alone.

Sentinel lymph node biopsy (SLNB) has become a standard component of melanoma management, and its 
prognostic and diagnostic benefits in adults are now wellunderstood $[3,4]$. However, only a few small studies have examined its efficacy and usefulness in the management of pediatric melanoma [5-8]. When SLNB is positive for spread of disease, the decision to perform complete lymph node dissection (CLND) is often undertaken [8]. With CLND comes an increased risk of morbidity. If benign pathology can be established at the outset, morbidity from wide resection SLNB and CLND can be prevented.

Recently, comparative genomic hybridization (CGH) has been described as a potential adjunct in the diagnosis of melanocytic lesions [9-13]. Based on known distinct DNA copy number changes in melanoma and nevi, CGH can be used to differentiate between malignant and benign lesions. CGH can be a useful diagnostic tool for histologically equivocal lesions and may prevent morbidity associated with more invasive procedures.

\section{Case report}

A 13-year-old Caucasian male presented to an outside physician for evaluation of an asymptomatic 4-mm uniformly pigmented nevus on the left supratip region of his nose. The lesion had been present since birth and had not changed in size, symmetry, or color. The patient admitted to frequent sun exposure but denied any history of blistering burns. There was no family history of cutaneous malignancy. His medical history and physical examination were otherwise unremarkable. Given the very low suspicion of malignancy, a shave biopsy was performed. Unexpectedly, pathologic examination revealed evidence of malignant melanoma involving the deep margins of the specimen. The patient was referred to the senior author (A.S.M.) for further evaluation (Fig. 1).

A metastatic workup revealed no evidence of lymphadenopathy or distant metastasis. A wide local excision with $1-\mathrm{cm}$ margins was performed under general anesthesia, and the defect was reconstructed with a full-thickness skin graft (Fig. 2). Histopathologic examination of the specimen demonstrated a proliferation of single and nested melanocytes involving both the epidermis and dermis. These cells were monomorphous with oval vesicular nuclei with both pale and pigmented cytoplasm. There was maturation with descent into the dermis. Dermal melanocytes tended to aggregate around pilosebaceous units. A few mitotic figures were limited to cells within the papillary dermis. In the epidermis, there was some evidence of pagetoid spread, with some single melanocytes reaching the cornified layer. Some of these cells were dendritic with pigmented cytoplasm and none were markedly atypical. Distinct uniform nucleoli were seen in dermal and epidermal melanocytes. Evaluation by the home institution's pathologist and by consultant dermatopathologists at two separate academic tertiary medical centers

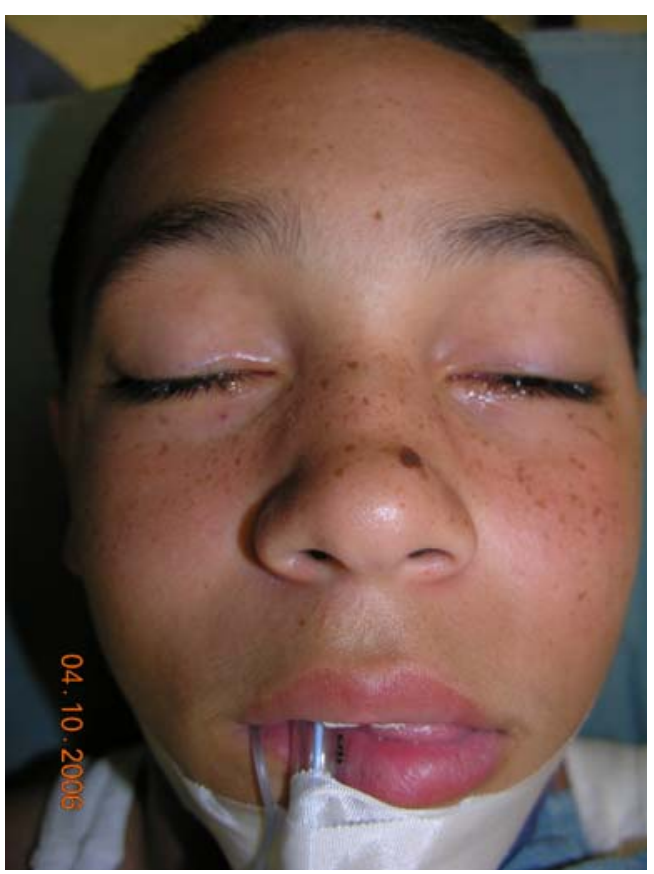

Fig. 1 The pigmented lesion is on the left supratip region of the nose

revealed the final diagnosis of malignant melanoma with a depth of $0.92 \mathrm{~mm}$.

The patient underwent sentinel lymph node biopsy after confirmation of the diagnosis 2 months after the initial shave biopsy. Initial lymphoscintigraphy of the face and neck was performed. Intra-operatively, a single left jugulodigastric lymph node was identified by gamma probe

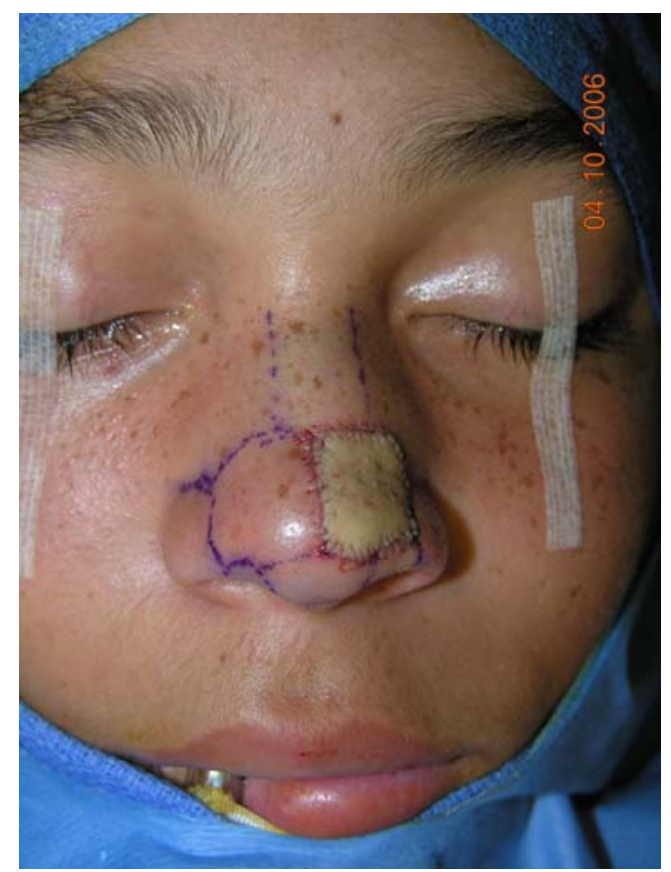

Fig. 2 A wide local excision with a 1-cm margin was performed, and the defect was reconstructed with a full-thickness skin graft 
detection and positive staining with Lymphazurin blue dye. Histopathologic evaluation of the lymph node revealed a single intracapsular focus of cells that were positive for Melan-A. This focus was limited to a few single cells clustered around a lymphatic one-cell stained positively for HMB-45. Histologically, these cells resembled cells from the primary lesion, and this was diagnosed as a small focus of metastatic melanoma. Based on these findings, the decision was made to perform a left-sided modified radical neck dissection approximately 1 month later. During this procedure, 53 lymph nodes from all five levels and the left submandibular gland were removed. The spinal accessory nerve, sternocleidomastoid muscle, jugular vein, and parotid gland were spared, and the operation was uncomplicated. All 53 nodes and the submandibular gland were negative for metastatic disease. The patient experienced no post-operative complications other than a minor decrease in neck range-of-motion which resolved with physical therapy.

Following the neck dissection, the patient's oncologist recommended a 1-year course of interferon alpha- $2 b$ therapy. Because of the associated toxicities of high-dose interferon therapy, the patient's parents desired further pathologic consultation before proceeding with treatment. At that time, two expert dermatopathologists independently reviewed the case and disagreed with the diagnosis of melanoma. Instead, each favored a diagnosis of benign nevus. Based on these conflicting opinions, the decision was made to proceed with CGH to better support one single diagnosis. Fixed tissue from both the shave and excision biopsies was sent for evaluation. $\mathrm{CGH}$ demonstrated a slight increase in tumor chromosome 16p profiles. However, the tumor:reference signal intensity ratio was less than 1.2, which was used as a cut-off for a true amplification. Furthermore, chromosome $16 \mathrm{p}$ has been found to be particularly susceptible to labeling artifacts which may lead to false-positive increases in signal intensity [14]. Thus, the increase in 16p was not considered to be abnormal. No other chromosomal aberrations were detected. These findings were consistent with a benign nevus rather than malignant melanoma. Based on the corroboration of the latest histopathologic evaluation and results of $\mathrm{CGH}$, the diagnosis was amended to compound melanocytic nevus of the nose. Additionally, the sentinel lymph node lesion was reclassified as a benign nevus cell rest.

\section{Discussion}

Melanoma comprises only 1-3\% of all childhood cancers [5-7]. Additionally, a subset of benign childhood melanocytic lesions such as Spitz nevi and certain congenital nevi may mimic melanoma, potentially delaying intervention. Even when such lesions are evaluated histologically, it is often difficult to differentiate between benign nevi and malignant melanoma.

The case presented illustrates this diagnostic challenge. Clinically, the patient's lesions had characteristics consistent with a benign etiology: it was present since birth, had not changed in size or color, and was asymptomatic. Additionally, the patient was an adolescent male and there was no family history of melanoma. Histologically, there were features favoring both benign (uniform cellular morphology, lack of atypia, and maturation with decent) and malignant (pagetoid spread and mitotic figures) etiologies, causing disagreement between pathologists. In these cases, there is a need to develop and implement the use of new diagnostic tools that can serve as adjuncts to histopathologic evaluation.

CGH is a relatively new technique used to scan the entire genome for chromosomal aberrations which are represented by DNA copy number changes [10]. This technique utilizes tissue from the tumor specimen as well as from a normal human control specimen. The samples are labeled with different fluorescent probes and then hybridized onto metaphase spreads. DNA from the two samples competed for chromosomal substrates. Based on measurements of tumor-to-control fluorescence intensity, one can determine whether DNA copy numbers in the tumor sample are increased, decreased, or equivalent compared to those in the control sample. Therefore, amplifications and deletions of different chromosomal segments can be detected in the tumor cells. Newer microarray-based methods allow for maximum resolution and can detect even single copy changes. One particular advantage of $\mathrm{CGH}$ is the ability to micro-dissect tissue from samples that have already been fixed [9]. Disadvantages include expense and the length of time needed for the procedure.

As a research tool, CGH has been used to identify distinct copy number changes in malignant melanoma and benign melanocytic nevi [10-13, 15]. Bastian et al. [11] demonstrated that while greater than $96 \%$ of malignant melanoma samples demonstrated chromosomal aberrations, most benign nevi did not. Aberrations most commonly found in melanoma samples were gains of chromosomes $6 \mathrm{p}, 1 \mathrm{q}, 7 \mathrm{p}, 7 \mathrm{q}, 8 \mathrm{q}, 17 \mathrm{q}$, and 20q; and losses of 9p, 9q, 10q, 10p, 6q, and 11q. Interestingly, while most benign nevi show no aberrations, Spitz nevi tend to demonstrate isolated gains of $11 \mathrm{p}$, a change that is not typically observed in malignant melanoma [10-12]. Benign proliferations arising within congenital melanocytic nevi (CMN) have also been found to have chromosomal aberrations [13]. However, these differ from aberrations found in malignant melanomas arising from $\mathrm{CMN}$, which are similar to those found in melanomas that arise de novo. While sample sizes used in these studies are relatively small, the results are promising and highlight the potential role of $\mathrm{CGH}$ as a tool to aid in the differentiation of benign and malignant melanocytic lesions. 
At present, the use of CGH in the clinical setting has been limited to a small number of cases. Its utility in confirming the histologic diagnosis of a benign nodule developing within a $\mathrm{CMN}$ in an infant was documented in a recent case report by Murphy et al. [9]. In the case of our 13-year-old patient, the use of CGH has important implications. Currently, little data exists regarding the usefulness of SLNB in malignant melanomas less than $1.0 \mathrm{~mm}$ deep, particularly in children. Such lesions may pose clinical decision-making challenges, especially when evidence of malignancy is not entirely certain based on histology. Furthermore, while SLNB is a useful tool for detecting nodal metastases, it does not always differentiate between malignant and benign lesions. Benign nevus-cell aggregates in lymph nodes are known to occur with benign primary lesions and in up to $22 \%$ of malignant melanomas [16, 17]. Any of these factors may have contributed to our patient's unnecessary surgical interventions. Fortunately, the patient did not experience any of the significant morbidities associated with CLND. However, had CGH been utilized earlier in the diagnostic process, the patient may have been able to avoid SLNB and CLND altogether. Thus, in cases where there is such uncertainty regarding diagnosis and treatment, the authors propose the use of CGH to support tissue diagnosis and to guide treatment plans.

\section{Conclusion}

The uncertain nature of a subset of melanocytic nevi, based on histology alone, warrants the development and implementation of better adjunctive diagnostic tools. As a research tool, CGH has identified several distinct differences in DNA copy number changes in malignant melanoma and benign nevi. Based on these known differences, CGH can serve as a potentially useful diagnostic tool in the clinical setting when lesions demonstrate equivocal histology. By helping to support histologic diagnoses, CGH may allow certain patients to avoid unnecessary invasive procedures such as CLND that are associated with significant morbidity. However, before the use of this method can become widespread, larger studies need to be conducted to better classify distinctions between malignant and benign melanocytic lesions.

Acknowledgment The authors would like to thank Dr. Philip LeBoit and colleagues at the UCSF Comprehensive Cancer Center for their diagnostic expertise and technical assistance.

\section{Conflicts of interest None}

\section{Financial disclosures None}

Open Access This article is distributed under the terms of the Creative Commons Attribution Noncommercial License which per- mits any noncommercial use, distribution, and reproduction in any medium, provided the original author(s) and source are credited.

\section{References}

1. Lodha S, Saggar S, Celebi JT, Silvers DN (2008) Discordance in the histopathologic diagnosis of difficult melanocytic neoplasms in the clinical setting. J Cutan Pathol 35:349-352

2. Wechsler J, Bastuji-Garin S, Spatz A et al (2002) Reliability of the histopathologic diagnosis of malignant melanoma in childhood. Arch Dermatol 138:625-628

3. Morton DL, Thompson JF, Cochran AJ et al (2006) Sentinel node biopsy or nodal observation in melanoma. N Engl J Med 355 (13):1307-1317

4. Morton DL, Cochran AJ, Thompson JF et al (2005) Sentinel node biopsy for early stage melanoma: accuracy and morbidity in MSLT-I, an international multicenter trial. Ann Surg 242:302-313

5. Pacella SJ, Lowe L, Bradford C, Marcus BC, Johnson T, Rees R (2003) The utility of sentinel lymph node biopsy in head and neck melanoma in the pediatric population. Plast Reconstr Surg 112:1257-1265

6. Roaten JB, Partrick DA, Bensard D, Pearlman N, Gonzalez R, Fitzpatrick J, McCarter MD (2005) Survival in sentinel lymph node-positive pediatric melanoma. J Pediatr Surg 40:988-992

7. Shah NC, Gerstle JT, Stuart M, Winter C, Pappo A (2006) Use of sentinel lymph node biopsy and high-dose interferon in pediatric patients with high-risk melanoma: the Hospital for Sick Children experience. J Pediatr Hematol Oncol 28(8):496-500

8. Dewar DJ, Newell B, Green MA, Topping AP, Powell BWEM, Cook MG (2004) The microanatomic location of metastatic melanoma in sentinel lymph nodes predicts nonsentinel lymph node involvement. J Clin Oncol 22:3345-3349

9. Murphy MJ, Jen M, Chang MW, Grant-Kels JM, Makkar H (2008) Molecular diagnosis of a benign proliferative nodule developing in a congenital melanocytic nevus in a 3-month-old infant. J Am Acad Dermatol 59:518-523

10. Bauer J, Bastian BC (2006) Distinguishing melanocytic nevi from melanoma by DNA copy number changes: comparative genomic hybridization as a research and diagnostic tool. Dermatologic Therapy 19:40-49

11. Bastian BC, Olshen AB, LeBoit PE, Pinkel D (2003) Classifying melanocytic tumors based on DNA copy number changes. Am J Pathol 163:1765-1770

12. Bastian BC (2003) Understanding the progression of melanocytic neoplasia using genomic analysis: from fields to cancer. Oncogene 22:3081-3086

13. Bastian BC, Xiong J, Frieden IJ, Williams ML, Chou P, Busam K, Pinkel D, LeBoit PE (2002) Genetic changes in neoplasms arising in congenital melanocytic nevi: differences between nodular proliferations and melanomas. Am J Pathol 161(4):1163-1169

14. Kallioniemi A, Kallioniemi OP, Piper J, Isola J, Waldman F, Gray JW, Pinkel D (1994) Optimizing comparative genomic hybridization for analysis of DNA sequence copy number changes in solid tumors. Genes Chromosomes Cancer 10:231-243

15. Carlson JA, Ross JS, Sliminski A, Linette G, Mysliborski J, Hill J, Mihm M (2005) Molecular diagnostics in melanoma. J Am Acad Dermatol 52:743-775

16. Zaharopoulos P, Hudnall SD (2004) Nevus-cell aggregates in lymph nodes: fine-needle aspiration cytologic findings and resulting diagnostic difficulties. Diagn Cytopathol 31:180-184

17. Biddle DA, Evans HL, Kemp BL, El-Naggar AK, Harvell JD, White WL, Iskandar SS, Prieto VG (2003) Intraparenchymal nevus cell aggregates in lymph nodes: a possible diagnostic pitfall with malignant melanoma and carcinoma. Am J Surg Pathol 27(5):673-681 